PROCEEDINGS OF THE

AMERICAN MATHEMATICAL SOCIETY

Volume 134, Number 4, Pages 921-929

S 0002-9939(05)08106-2

Article electronically published on July 19, 2005

\title{
DIVISION ALGEBRAS THAT RAMIFY ONLY ON A PLANE QUARTIC CURVE
}

\author{
BORIS È. KUNYAVSKIĬ, LOUIS H. ROWEN, SERGEY V. TIKHONOV, \\ AND VYACHESLAV I. YANCHEVSKII
}

(Communicated by Martin Lorenz)

\begin{abstract}
Let $k$ be an algebraically closed field of characteristic 0 . We prove that any division algebra over $k(x, y)$ whose ramification locus lies on a quartic curve is cyclic.
\end{abstract}

\section{MAin RESUlt}

Let $k$ be an algebraically closed field of characteristic 0 , with $L=k(x, y)$ its purely transcendental extension of degree 2 . We are interested in division $L$-algebras with ramification only at a quartic curve. Our main result (Theorem 1.2), which grew out from our earlier paper [11, shows that any such algebra is a symbol algebra whose index equals the exponent. This is a generalization of earlier results of Ford, Van den Bergh and Jacob on cyclicity of $k(x, y)$-algebras ramified either along a cubic curve, or a hyperelliptic curve, or a special quartic curve (see [5], [6], 7, 9], 16]). In contrast to the above cited papers (and to the paper of de Jong [10] who proved the equality of the index and the exponent for any central simple algebra defined over an extension of $k$ of transcendence degree 2, regardless of its ramification locus), our methods are quite elementary and describe the cyclic algebra explicitly in terms of the ramification. This might be a justification for publishing this result, in spite of the fact that the long-standing problem of cyclicity of $k(x, y)$-algebras seems to be close to a general solution by M. Ojanguren and R. Parimala (private communication of Parimala to one of the authors). Roughly, our approach consists of regarding $L$ as the function field $l\left(\mathbb{P}_{l}^{1}\right)$ of the projective line over $l=k(x)$ (rather than the function field $k\left(\mathbb{P}_{k}^{2}\right)$ of the projective plane over $k$ ). As observed by the anonymous referee of [11, this allows one to apply the full strength of the Faddeev theory of algebras over a projective line (and, in particular, to employ classification results of [1] for algebras with small ramification locus).

Received by the editors October 20, 2004.

2000 Mathematics Subject Classification. Primary 16K20.

The first author was partially supported by the Ministry of Absorption (Israel) and the Minerva Foundation through the Emmy Noether Research Institute of Mathematics.

The third and the fourth authors were partially supported by the Fundamental Research Foundation of Belarus.

This research was supported by the Israel Science Foundation founded by the Israel Academy of Sciences and Humanities - Center of Excellence Program and by RTN Network HPRN-CT2002-00287.

(C)2005 American Mathematical Society Reverts to public domain 28 years from publication 
We fix an integer $n>1$. Let $\operatorname{Br}(F)$ denote the Brauer group of a field $F$ and ${ }_{n} \operatorname{Br}(F)$ its $n$-torsion subgroup. Assume $F$ contains a primitive $n$th root of unity $\rho$ whose notation is fixed. For any finite-dimensional $F$-central simple algebra $\mathcal{A}$, we use $[\mathcal{A}]$ to denote its class in $\operatorname{Br}(F)$. The exponent $\exp (\mathcal{A})$ of $\mathcal{A}$ is the order of $[\mathcal{A}]$ in $\operatorname{Br}(F)$, and the index $\operatorname{ind}(\mathcal{A})$ of $\mathcal{A}$ is the degree of the central division algebra $\mathcal{D}$ such that $\mathcal{A} \cong M_{r}(\mathcal{D})$. If $a, b \in F^{*}$, we denote by $(a, b)_{n}$ the cyclic algebra generated over $F$ by $\alpha, \beta$ such that $\alpha^{n}=a, \beta^{n}=b, \alpha \beta=\rho \beta \alpha$. (We often drop the subscript $n$ if this does not lead to any confusion.)

Let $R$ be a discrete valuation ring with quotient field $K$ and residue field $l$. We assume that both $l$ and $K$ are of characteristic zero. Then it is classical 2 that there is an exact sequence

$$
0 \rightarrow \operatorname{Br}(R) \rightarrow \operatorname{Br}(K) \stackrel{\text { ram }}{\longrightarrow} H^{1}(l, \mathbb{Q} / \mathbb{Z}) \rightarrow 0 .
$$

The ramification map, denoted by ram, is described in [2] (see also [14, Ch.10]).

Now let $k$ be an algebraically closed field of characteristic zero, and let $L=k\left(\mathbb{P}_{k}^{2}\right)$ be the function field of the projective plane over $k$.

Let $C \subset \mathbb{P}_{k}^{2}$ be an irreducible curve. The local ring $\mathcal{O}_{C}$ is a discrete valuation ring, and we denote by $v_{C}: L^{*} \rightarrow \mathbb{Z}$ the corresponding discrete valuation.

We view $\mathbb{P}_{k}^{2}=\operatorname{Proj}(k[x, y, z])$ as $\operatorname{Spec}(k[x, y]) \cup\left\{L_{\infty}\right\}$ where $L_{\infty}$ is the infinite line corresponding to $z=0$. (Abusing notation, we write $k[x, y]$ for what really should be $k[(x / z),(y / z)]$.) Except for $L_{\infty}$, each $C$ corresponds to an irreducible polynomial in either $k(x)[y]$ or $k(y)[x]$, and the valuation $v_{C}$ comes from the localization at that polynomial. At $L_{\infty}$, we must use the $1 / x$-adic valuation on $k(x / y)[x]$ to compute $v_{L_{\infty}}$.

Then there is the map corresponding to $\mathcal{O}_{C}$ :

$$
\operatorname{ram}_{C}: \operatorname{Br}(L) \rightarrow H^{1}(k(C), \mathbb{Q} / \mathbb{Z}),
$$

where $k(C)$ is the function field of $C$.

Note that ${ }_{n} H^{1}(k(C), \mathbb{Q} / \mathbb{Z}) \cong k(C)^{*} / k(C)^{* n}$. By the "tame symbol" formula (see, for example, [3, 1.3 and 2.3]), the ramification of $\left[(a, b)_{n}\right]$ is defined by the $n$th root of the residue of

$$
(-1)^{v_{C}(a) v_{C}(b)} a^{v_{C}(b)} / b^{v_{C}(a)}
$$

According to 1 , there is a monomorphism $\operatorname{Br}(L) \rightarrow \bigoplus H^{1}(k(C), \mathbb{Q} / \mathbb{Z})$, where $C$ runs over all irreducible curves in $\mathbb{P}_{k}^{2}$; this map will also be denoted by ram. For $[\mathcal{A}] \in \operatorname{Br}(L)$ we write

$$
R=\bigcup_{C}\left\{C \subset \mathbb{P}_{k}^{2} \mid \operatorname{ram}_{C}([\mathcal{A}]) \neq 0\right\}
$$

and call $R$ the ramification divisor of $\mathcal{A}$.

Let $l$ be a field of characteristic zero. According to [4, 15, Ch. II, App., §5], there is an exact sequence

$$
0 \rightarrow{ }_{n} \operatorname{Br}(l) \rightarrow{ }_{n} \operatorname{Br}\left(l\left(\mathbb{P}_{l}^{1}\right)\right) \stackrel{\oplus \partial_{M}}{\longrightarrow} \bigoplus_{M \in \mathbb{P}_{l}^{1}} H^{1}(l(M), \mathbb{Z} / n) \stackrel{\oplus \operatorname{cor}_{M}}{\longrightarrow} H^{1}(l, \mathbb{Z} / n) \rightarrow 0 .
$$

Here $M$ runs over the closed points of $\mathbb{P}_{l}^{1}$, and $\oplus \operatorname{cor}_{M}$ is the sum of corestriction homomorphisms $\operatorname{cor}_{M}: H^{1}(l(M), \mathbb{Z} / n) \longrightarrow H^{1}(l, \mathbb{Z} / n)$. Moreover, using the isomorphism $H^{1}(l(M), \mathbb{Z} / n) \cong l(M)^{*} /\left(l(M)^{*}\right)^{n}$ one can compute cor $_{M}$ by the formula

$$
\operatorname{cor}_{M}\left(a\left(l(M)^{*}\right)^{n}\right)=N_{l(M) / l}(a)\left(l^{*}\right)^{n} .
$$


Sequence (1.1) is sometimes referred to as the Faddeev reciprocity law; indeed, it says that the sum of corestrictions of the residues of a given algebra is zero.

Two $l(t)$-algebras $\mathcal{A}$ and $\mathcal{B}$ are said to be Faddeev equivalent (our notation for that is $\mathcal{A} \sim \sim_{\mathcal{F}} \mathcal{B}$ ) if there exists an $l$-algebra $\mathcal{C}$ such that $\mathcal{A}$ is Brauer equivalent to $\mathcal{B} \otimes\left(\mathcal{C} \otimes_{l} l(t)\right)$. By the reciprocity law, two algebras having the same residues in all but one closed rational point of ramification must have the same residue in that point as well and are thus Faddeev equivalent. In particular, two algebras having the same residues in all finite points are Faddeev equivalent.

Remark 1.1. In case $\operatorname{Br}(l)$ is trivial, Faddeev equivalent algebras are Brauer equivalent. This is the case when $l=k(x)$ for $k$ algebraically closed, by Tsen's theorem.

There is a one-to-one correspondence between closed points of $\mathbb{P}_{l}^{1}$ and discrete valuations of $l\left(\mathbb{P}_{l}^{1}\right)=l(t)$ trivial on $l$. Recall the structure of discrete valuations of $l(t)$ trivial on $l$. Any such valuation is of the following form. If it corresponds to a finite closed point $M$ of $\mathbb{P}_{l}^{1}$, then there exists an irreducible monic polynomial $f(t) \in l[t]$ such that the valuation $v_{f}$ with $f(t)$ as a uniformizer coincides with the valuation corresponding to $M$. The valuation corresponding to the infinite point is $v_{\infty}$ with $t^{-1}$ as a uniformizer.

Let $f(t), g(t) \in l[t], f$ irreducible and not dividing $g$. Then the ramification of $(g(t), f(t))_{n}$ at $f$ is $l(\theta)(\sqrt[n]{g(\theta)}) / l(\theta)$ (here and throughout below we identify elements of $l(\theta)^{*} / l(\theta)^{* n}$ with degree $n$ cyclic extensions of $\left.l(\theta)\right)$.

Let $F(x, y) \in k[x, y]$ be an irreducible polynomial, $F(x, y)=\sum_{i=0}^{n} g_{i}(x) y^{i}$, where $g_{i}(x) \in k[x], g_{n}(x) \neq 0$. Let $C$ be the curve defined by the equation $F(x, y)=$ 0 . Assume that $n>0$. As mentioned above, the field $k\left(\mathbb{P}_{k}^{2}\right)=k(x, y)$ can be interpreted as the function field $k(x)\left(\mathbb{P}_{k(x)}^{1}\right)=k(x)(y)$ of the line over the field $k(x)$.

Let $f(y)=F(x, y) / g_{n}(x) \in k(x)[y]$. Then $f(y)$ is a monic irreducible polynomial over $k(x)$, and the valuation of $k(x, y)$ corresponding to the curve $C$ coincides with the valuation of $k(x)(y)$ corresponding to the closed point defined by the polynomial $f(y)$.

Thus, a central simple algebra $\mathcal{A} / k\left(\mathbb{P}_{k}^{2}\right)$ ramifies at the valuation corresponding to the curve $C$ if and only if $\mathcal{A} / k(x)\left(\mathbb{P}_{k(x)}^{1}\right)$ ramifies at a closed point defined by the polynomial $f(y)$.

Note that if $n=0$ (i.e., $F(x, y)=x+c$, where $c \in k$ ), then the valuation corresponding to the curve $C$ is not trivial on $k(x)$. Hence in this case there is no valuation of $k(x)(y)$ corresponding to the valuation of $k(x, y)$ defined by $C$. Moreover, an algebra $\mathcal{A}$, viewed as an algebra over $k(x)\left(\mathbb{P}_{k(x)}^{1}\right)=k(x)(y)$, may ramify at the infinite point even if $\mathcal{A}$, viewed as an algebra over $k\left(\mathbb{P}_{k}^{2}\right)$, does not ramify at the infinite line $L_{\infty}$ given by $z=0$. For example, consider the quaternion algebra $\mathcal{B}=\left(x y, x^{2}+1\right)$ [11. It does not ramify at the infinite line because the valuation $v_{L_{\infty}}$ (corresponding to the infinite line) of both $x y$ and $x^{2}+1$ equals -2 . Indeed, $v_{L_{\infty}}$ coincides with the valuation $v_{\infty}$ of $k(x / y)(x)$ corresponding to the infinite point. Hence we have

$$
v_{L_{\infty}}(x y)=v_{\infty}(x y)=v_{\infty}\left(x^{2} y / x\right)=-2
$$

since $y / x$ is "constant". Similarly, $v_{L_{\infty}}\left(x^{2}+1\right)=v_{\infty}\left(x^{2}+1\right)=-2$.

On the other hand, as an algebra over $k(x)\left(\mathbb{P}_{k(x)}^{1}\right), \mathcal{B}$ ramifies at the infinite point because $x^{2}+1 \notin k(x)^{2}$. 
We are now ready to formulate our main result.

Theorem 1.2. Let $k$ be an algebraically closed field of characteristic zero, and let $\mathcal{D}$ be a division algebra over $L=k(x, y)=k\left(\mathbb{P}_{k}^{2}\right)$ with ramification only along a quartic curve $C \subset \mathbb{P}_{k}^{2}$. Then $\mathcal{D}$ is a cyclic algebra, and $\exp (\mathcal{D})=\operatorname{ind}(\mathcal{D})$.

The general strategy of the proof is as follows. As mentioned above, we view $L$ as the function field $l\left(\mathbb{P}_{l}^{1}\right)$, where $l=k(x)$. Then we can use the following key result describing central simple $k(x)$-algebras with small ramification locus up to Faddeev equivalence:

Proposition 1.3. Let $K$ be an arbitrary field of characteristic zero, containing the $n$th roots of unity, $F=K(t), \mathcal{A}$ a central simple $F$-algebra of exponent $n$. Suppose that $\mathcal{A}$ has ramification either

(i) at most at three linear points, or

(ii) at a point corresponding to a quadratic irreducible polynomial, and perhaps at infinity, or

(iii) at a point corresponding to a cubic irreducible polynomial.

Then $\mathcal{A}$ is Faddeev equivalent to a cyclic F-algebra of degree $n$.

Remark 1.4. Cases (i) and (ii) are none other than Propositions 2.2 and 2.3 of [11] (stated in a slightly different form); for the sake of completeness and the reader's convenience we reproduce the proofs in Section 2 below. We did not manage to treat case (iii) in [11] and fill this gap here (for the case $n=2$ this was done in [12]).

We then continue the proof considering, case by case, all possible configurations of irreducible components of the ramification divisor $C$ :

(i) $C$ is a union of four lines;

(ii) $C$ is a union of two lines and an irreducible conic;

(iii) $C$ is a union of two irreducible conics;

(iv) $C$ is a union of a line and an irreducible cubic curve;

(v) $C$ is irreducible.

In each case we show that by a projective transformation of the plane one can bring a given algebra $\mathcal{D}$ to the required form:

Proposition 1.5. Let $k$ be an algebraically closed field of characteristic zero, $\mathcal{A}$ an algebra over $k\left(\mathbb{P}^{2}\right)=k(x, y)$ with ramification at a plane quartic $C$. Then, after a projective transformation of $\mathbb{P}^{2}, \mathcal{A}$ viewed as an algebra over $L=l(y)$, where $l=k(x)$, satisfies the hypotheses of Proposition 1.3 ,

Proof of Theorem 1.2 from Propositions 1.3 and 1.5, $\mathcal{D}$ is Faddeev equivalent and thus, by Remark 1.1. Brauer equivalent to a cyclic algebra $\mathcal{C}$ of degree $n$. Hence $\operatorname{ind}(\mathcal{C}) \mid n$. But $n=\exp (\mathcal{C}) \mid \operatorname{ind}(\mathcal{C})$, implying $\operatorname{ind}(\mathcal{C})=n=\operatorname{deg}(\mathcal{C})$. Hence $\mathcal{C}$ is a division algebra, so $\mathcal{D} \approx \mathcal{C}$ and $\operatorname{ind}(\mathcal{D})=\exp (\mathcal{D})$.

\section{Proof of Proposition 1.3}

Recall that if two algebras have the same ramification at all finite points, then they have the same ramification at infinity, by Faddeev's reciprocity law, and thus are Faddeev equivalent. 
(i) Using the automorphism group of $\mathbb{P}_{K}^{1}$, we may assume that the points of ramification of $\mathcal{A}$ are $t, t-1$, and $\infty$ with ramification $K\left(\sqrt[n]{b_{1}}\right) / K, K\left(\sqrt[n]{b_{2}}\right) / K$, and $K\left(\sqrt[n]{b_{3}}\right) / K$ respectively.

The cyclic algebra $\mathcal{B}=\left(b_{2} t,-b_{1}^{-1}(t-1)\right)$ has prescribed ramification at $t$ and $t-1$. Hence $\mathcal{A} \sim_{\mathcal{F}} \mathcal{B}$.

(ii) Let the ramification of $\mathcal{A}$ at a quadratic polynomial $f$ be given by a cyclic extension $K(\theta)(\sqrt[n]{u+v \theta}) / K(\theta)$, where $\theta$ is a root of $f, u, v \in K$.

First suppose $v=0$. Consider the algebra $\mathcal{B}=(u, f)_{n}$. It is ramified at $f$ and perhaps at infinity. Its ramification at $f$ coincides with the ramification of $\mathcal{A}$ at $f$. Hence $\mathcal{A} \sim \mathcal{F} \mathcal{B}$.

Now suppose $v \neq 0$. Denote by $c_{u v}$ the value of $f(t)$ at $-u / v$. We prove that $\mathcal{A}$ is Faddeev equivalent to $\mathcal{B}=\left(u+v t, f(t) / c_{u v}\right)$. Indeed, $\mathcal{B}$ could only ramify at $f, t+u / v$, and infinity, but we see that $\mathcal{B}$ is unramified at $t+u / v$ since $f(-u / v) / c_{u v}=1$. It has the same ramification at $f$ as $\mathcal{A}$ and thus $\mathcal{A} \sim_{\mathcal{F}} \mathcal{B}$.

(iii) Assume $\mathcal{A}$ has ramification $K(\sqrt[n]{a}) / K$ at $f$, where $a \in K(\theta)$ and $\theta$ is a root of $f$. Then $a=a_{0}+a_{1} \theta+a_{2} \theta^{2}$. We shall consider separately three cases.

1. $a_{1}=a_{2}=0$. Then the algebra $\left(f(t), a_{0}\right)$ has at $f$ the same ramification as $\mathcal{A}$, so is Faddeev equivalent to $\mathcal{A}$.

2. $a_{2}=0, a_{1} \neq 0$. Consider the algebra

$$
\mathcal{B}=\left(f(t) / f\left(-a_{0} / a_{1}\right), a_{0}+a_{1} t\right) .
$$

The algebra $\mathcal{B}$ has at $f$ the same ramification as $\mathcal{A}$. Moreover, as in (ii), $\mathcal{B}$ has no ramification at $a_{0}+a_{1} t$. Hence $\mathcal{A} \sim_{\mathcal{F}} \mathcal{B}$.

3. $a_{2} \neq 0$. First, let us show that there exists $c \in K$ such that $a=\left(b_{0}+b_{1} \theta\right) /$ $(c+\theta)$ for some $b_{0}, b_{1} \in K$.

Let $f(t)=t^{3}-m_{2} t^{2}-m_{1} t-m_{0}$. We have $a(c+\theta)=a_{2} \theta^{3}+\left(a_{2} c+a_{1}\right) \theta^{2}+$ terms of lower degree in $\theta$. But $\theta^{3}=m_{2} \theta^{2}+m_{1} \theta+m_{0}$, so setting $c=-\left(m_{2}+a_{1} / a_{2}\right)$ yields the $\theta^{2}$-term $=a_{2} m_{2} \theta^{2}+a_{2} c \theta+a_{1} \theta^{2}=0$, whence $a(c+\theta)=b_{0}+b_{1} \theta$ and $a=\left(b_{0}+b_{1} \theta\right) /(c+\theta)$.

Consider two subcases:

a) Let $b_{1} \neq 0$. Consider the algebra

$$
\left.\mathcal{C}_{1}=\left((c+t)^{2 n-3} f(t)\right) /\left(\left(c-b_{0} / b_{1}\right)^{2 n-3} f\left(-b_{0} / b_{1}\right)\right),\left(b_{0}+b_{1} t\right)(c+t)^{n-1}\right) .
$$

Then the algebra $\mathcal{C}_{1}$ has at $f$ the same ramification as $\mathcal{A}$ since

$\left(b_{0}+b_{1} \theta\right)(c+\theta)^{n-1} \equiv\left(b_{0}+b_{1} \theta\right)(c+\theta) \min (c+\theta)^{n} \equiv a(c+\theta)^{n} \equiv a \quad\left(\bmod \left(K(\theta)^{*}\right)^{n}\right)$.

Furthermore, since both sides of the symbol algebra $\mathcal{C}_{1}$ are polynomials of degree divisible by $n$, using the tame symbol formula we conclude that $\mathcal{C}_{1}$ has no ramification at infinity. Moreover, $\mathcal{C}_{1}$ has no ramification at $t+b_{0} / b_{1}$. By the reciprocity law, $\mathcal{C}_{1}$ has no ramification at $c+t$. Thus $\mathcal{A} \sim_{\mathcal{F}} \mathcal{C}_{1}$.

b) If $b_{1}=0$, then $\mathcal{A} \sim_{\mathcal{F}}\left(f(t) / f(-c), b_{0}(c+t)^{n-1}\right)$.

Remark 2.1. In each case in the proof of Proposition 1.3. we have provided an explicit cyclic algebra Faddeev equivalent to $\mathcal{A}$.

\section{Proof of Proposition 1.5}

(i) Suppose $C$ is the union of 4 lines. Using the automorphism group of $\mathbb{P}_{k}^{2}$, we may throughout assume that $\mathcal{A}$ ramifies at the infinite line and three finite lines. 
Moreover, we may and shall assume that one finite line is defined by the equation $x=0$. Let two other finite lines be defined by the equations

$$
a_{i} x+b_{i} y+c_{i}=0, i=1,2 .
$$

Hence the algebra $\mathcal{A}$, viewed as an algebra over $k(x)\left(\mathbb{P}_{k(x)}^{1}\right)$, ramifies at most at two linear polynomials and perhaps at the infinite point and thus satisfies the hypotheses of Proposition 1.3(i).

(ii) Suppose $C$ is the union of two lines and an irreducible conic. Using the automorphism group of $\mathbb{P}_{k}^{2}$ as in (i), we may assume that $\mathcal{A}$ ramifies at the infinite line, one finite line, and an irreducible conic. Moreover, we may and shall assume that the finite line is defined by the equation $x=0$. Let the conic be defined by the equation $F(x, y)=0$, where $\operatorname{deg} F(x, y)=2$. Hence the algebra $\mathcal{A}$, viewed as an algebra over $k(x)\left(\mathbb{P}_{k(x)}^{1}\right)$, ramifies at most at a quadratic polynomial and perhaps at the infinite point and thus satisfies the hypotheses of Proposition 1.3(ii).

(iii) Again, we may assume that $\mathcal{A}$ ramifies at the infinite line and an irreducible cubic defined by the equation

$$
a_{0} y^{3}+a_{1} y^{2} x+a_{2} y x^{2}+a_{3} x^{3}+g(x, y)=0,
$$

where $a_{i} \in k$ and $\operatorname{deg} g(x, y) \leq 2$.

Let $a \in k$ be such that $a_{0}+a_{1} a+a_{2} a^{2}+a_{3} a^{3}=0$. Then after the substitution $x=x^{\prime}+a y$ we may assume that the cubic defined by equation (3.1) satisfies $a_{0}=0$.

Hence the algebra $\mathcal{A}$, viewed as an algebra over $k(x)\left(\mathbb{P}_{k(x)}^{1}\right)$, ramifies at most at a quadratic polynomial in $y$ and perhaps at the infinite point, and we are done.

(iv) Suppose $C$ is the union of two irreducible conics defined by the homogeneous equations

$$
\begin{gathered}
a_{0} y^{2}+a_{1} y x+a_{2} x^{2}+a_{3} z^{2}+a_{4} x z+a_{5} y z=0, \\
b_{0} y^{2}+b_{1} y x+b_{2} x^{2}+b_{3} z^{2}+b_{4} x z+b_{5} y z=0,
\end{gathered}
$$

where $a_{i}, b_{i} \in k$. Since any two curves in the projective plane intersect, there exists a nontrivial solution $\left(u_{11}, u_{12}, u_{13}\right)$ of the following system of equations:

$$
\left\{\begin{array}{l}
a_{0} u_{22}^{2}+a_{1} u_{12} u_{22}+a_{2} u_{12}^{2}+a_{3} u_{32}^{2}+a_{4} u_{12} u_{32}+a_{5} u_{22} u_{32}=0 \\
b_{0} u_{22}^{2}+b_{1} u_{12} u_{22}+b_{2} u_{12}^{2}+b_{3} u_{32}^{2}+b_{4} u_{12} u_{32}+b_{5} u_{22} u_{32}=0 .
\end{array}\right.
$$

Hence there exist $u_{i j} \in k, i=2,3, j=1,2,3$, such that the matrix $M=\left(u_{i j}\right)_{i, j=1,2,3}$ is invertible. Applying the automorphism of $\mathbb{P}_{k}^{2}$ defined by $M^{-1}$, we obtain that the conics are defined by equations (3.2) and (3.3) with $a_{0}=b_{0}=0$.

Hence the algebra $\mathcal{A}$, viewed as an algebra over $k(x)\left(\mathbb{P}_{k(x)}^{1}\right)$, ramifies at most at two linear polynomials and perhaps at the infinite point and, as in (ii), satisfies the hypotheses of Proposition 1.3(ii).

(v) Suppose $C$ is an irreducible quartic.

We need some more notation. Let $X$ be a projective irreducible curve (not necessarily smooth), $P$ a point on $X, R=O_{X, P}$ the local ring of $X$ at $P$. For $f \in R$ one can define the order function $\operatorname{ord}_{P}(f)$ as $\operatorname{dim}_{k} R / f R$ (see [8, 1.2]), continue this function to $k(X)^{*}$, and show that $\operatorname{ord}_{P}: k(X)^{*} \rightarrow \mathbb{Z}$ is a homomorphism of abelian groups (see [8, A.3]).

Lemma 3.1. Let $C \subset \mathbb{P}_{k}^{2}$ be a projective curve. Suppose that an affine part $C^{\prime}=$ $C \cap \mathbb{A}_{k}^{2}$ of $C$ is given by an irreducible polynomial $F(x, y)$ such that $\operatorname{deg}_{y} F>0$ and 
$F$ is monic as a polynomial in $y$. Let $f \in k(C)^{*}$ be such that $n \mid \operatorname{ord}_{P}(f)$ for any $P \in C^{\prime}$. Then $N_{k(C) / k(x)}(f) \in\left(k(x)^{*}\right)^{n}$.

Proof. Consider the projection $\psi: C^{\prime} \rightarrow \mathbb{A}_{k(x)}^{1}$ corresponding to the homomorphism $k[x] \rightarrow k[x, y] \rightarrow k\left[C^{\prime}\right]=k[x, y] /(F)$. By hypothesis, $F$ is monic as a polynomial in $y$; hence the image of $y$ in $k\left[C^{\prime}\right]$ is integral over $k[x]$. Therefore the ring $k\left[C^{\prime}\right]$ is integral over $k[x]$, and thus $\psi$ is a finite morphism. Hence $\psi$ is proper and surjective, and by [8, Prop. 1.4(b)] we have

$$
\psi_{*}(\operatorname{div}(f))=\operatorname{div}\left(N_{k(C) / k(x)}(f)\right) .
$$

By hypothesis, all coefficients of $\operatorname{div}(f)$ at finite points $P$ are divisible by $n$. We conclude that the coefficients of the divisor in the right-hand side of (3.4) at all finite points are also divisible by $n$. This means that the values of the corresponding valuations are divisible by $n$, and since $k$ is algebraically closed, $N_{k(C) / k(x)}(f)$ is an $n$-th power.

We need another auxiliary result.

Lemma 3.2. Let $k$ be an algebraically closed field of characteristic zero, $l=k(x)$, $L=k\left(\mathbb{P}_{k}^{2}\right)=k(x, y)$, and $\mathcal{A}$ an algebra over $L$ with ramification at only one irreducible curve $C \subset \mathbb{P}^{2}$ satisfying the hypotheses of Lemma 3.1. Then $\mathcal{A}$ as an algebra over $l\left(\mathbb{P}_{l}^{1}\right)=k(x)(y)$ has no ramification at the infinite point.

Proof. Maintain the notation of Lemma 3.1. Denote $n=\exp (\mathcal{A})$. Suppose that the ramification of $\mathcal{A}$ at $C$ is defined by $f \in k(C)$. Note that $k(C)$ is the residue field corresponding to the valuation of $k(x, y)$ defined by $C$. According to [1] (see also [13, p.196] for a more general setting), there is an exact sequence

$$
0 \rightarrow \operatorname{Br}(L) \stackrel{\oplus \operatorname{ram}_{Y}}{\longrightarrow} \bigoplus_{Y \subset \mathbb{A}_{k}^{2(1)}} H^{1}(k(Y), \mathbb{Q} / \mathbb{Z}) \stackrel{\phi}{\longrightarrow} \bigoplus_{Q \in \mathbb{A}_{k}^{2(2)}} \mathbb{Q} / \mathbb{Z} \longrightarrow 0,
$$

where $Y$ and $Q$ range over all irreducible subvarieties of $\mathbb{A}_{k}^{2}$ of codimension 1 and 2, respectively. The map $\phi$ is defined as follows. Let $Q \in \mathbb{A}_{k}^{2(2)}, Y \in \mathbb{A}_{k}^{2(1)}$. Suppose $\alpha \in H^{1}(k(Y), \mathbb{Q} / \mathbb{Z})$ is of order $n$ and defined by $a \in k(Y)$. Assume $Q \in Y$. Set $r=\operatorname{ord}_{Q}(a)$ and define the ramification of $\alpha$ at $Q$ by $\operatorname{ram}_{Q}(\alpha)=r / n$.

If $Q$ is not a subset of $Y$, we set $\operatorname{ram}_{Q}(\alpha)=0$. The map $\phi$ is the sum of all maps $\operatorname{ram}_{Q}$.

Using sequence (3.5), we obtain $\operatorname{ram}_{P}\left(\operatorname{ram}_{C}([\mathcal{A}])\right)=0$ for any $P \in C^{\prime}$. Hence $n \mid \operatorname{ord}_{P}(f)$ for any $P \in C^{\prime}$. By Lemma 3.1, we have $N_{k(C) / k(x)}(f) \in\left(k(x)^{*}\right)^{n}$. Hence from the exact sequence (1.1) we obtain $\operatorname{cor}_{p}(f)=0$, where $p$ is the closed point of $\mathbb{P}_{k(x)}^{1}$ corresponding to the valuation of $k(x, y)$ defined by $C$. Thus $\mathcal{A}$ as an algebra over $l\left(\mathbb{P}_{l}^{1}\right)=k(x)(y)$ has no ramification at the infinite point.

We can now prove the assertion of case (v) of Proposition 1.5.

Without loss of generality we may assume that $\operatorname{deg}_{y} F>0$, for otherwise $\mathcal{A}$ is unramified over $k(x)\left(\mathbb{P}_{k(x)}^{1}\right)$. Moreover, we may assume $\operatorname{deg}_{y} F>2$, for otherwise we are reduced to Proposition 1.3((i) or (ii)). Furthermore, by an argument analogous to that in case (iii), we may and shall assume that the coefficient at $y^{4}$ is zero. Let us show that one can transform $C$ so that it satisfies the hypotheses of Lemma 3.1 . 
Write an affine equation for $C^{\prime}$ in the form

$$
y^{3}\left(a_{1} x+a_{0}\right)+y^{2} g_{2}(x)+y g_{3}(x)+g_{4}(x)=0,
$$

where $g_{i}(x) \in k[x]$ denotes a polynomial of degree $i(2 \leq i \leq 4)$. If $a_{1}=0$, on dividing by $a_{0}$ we arrive at the hypotheses of Lemma 3.1. So suppose $a_{1} \neq 0$ and consider the homogeneous equation of $C$ :

$$
y^{3}\left(a_{1} x+a_{0} z\right)+y^{2} G_{2}(x, z)+y G_{3}(x, z)+G_{4}(x, z)=0,
$$

where $G_{i}(x, z)$ stands for the homogenization of $g_{i}(x)$. The projective transformation of the plane $x^{\prime}=a_{1} x+a_{0} z, y=y, z=z$ brings $C$ to the form

$$
y^{3} x^{\prime}+y^{2} H_{2}\left(x^{\prime}, z\right)+y H_{3}\left(x^{\prime}, z\right)+H_{4}\left(x^{\prime}, z\right)=0,
$$

where $H_{i}$ is homogeneous of degree $i$. Going over to another affine chart on dividing by $x^{\prime 4}$, we obtain an equation for another affine part of $C$ which satisfies the hypotheses of Lemma 3.1 .

We can now apply Lemma 3.2 to conclude that the algebra $\mathcal{A}$, viewed as an algebra over $k(x)\left(\mathbb{P}_{k(x)}^{1}\right)$, ramifies only at a cubic irreducible polynomial, the hypothesis of Proposition 1.3(iii).

Proposition 1.5 is proved. This completes the proof of Theorem 1.2 .

\section{REFERENCES}

[1] M. Artin and D. Mumford, Some elementary examples of unirational varieties which are not rational, Proc. London Math. Soc. (3) 25 (1972), 75-95. MR0321934 (48:299)

[2] M. Auslander and A. Brumer, Brauer groups of discrete valuation rings, Nederl. Akad. Wetensch. Proc., Ser. A 71 (1968), 286-296. MR0228471 (37:4051)

[3] S. Bloch, Torsion algebraic cycles, $K_{2}$, and Brauer groups of function fields, "Groupe de Brauer" (Sém., Les Plans-sur-Bex, Suisse 1980, M. Kervaire and M. Ojanguren, Eds.), Lecture Notes Math., vol. 844, Springer, Berlin-Heidelberg-New York, 1981, pp. 75-102. MR0611866 (82k:14018)

[4] D. K. Faddeev, Simple algebras over a function field in one variable, Proc. Steklov Inst. 38 (1951), 321-344; English transl. in AMS Transl. 3 (1956), 15-38. MR0077505 (17:1046e)

[5] T. J. Ford, Division algebras that ramify along a singular plane cubic curve, New York J. Math. 1 (1995), 178-183. MR:1348596 (96i:12002)

[6] T. J. Ford, Division algebras that ramify only on a plane quartic curve with simply connected components, Algebr. Represent. Theory 6 (2003), 501-514. MR2026724 (2004k:16042)

[7] T. J. Ford, Division algebras that ramify only on a plane nodal cubic curve plus a line, J. Pure Appl. Algebra 188 (2004), 117-126. MR2030809 (2004k:16043)

[8] W. Fulton, Intersection Theory, Springer-Verlag, Berlin et al., 1984. MR.0732620|(85k:14004)

[9] B. Jacob, Division algebras which only ramify along a hyperelliptic curve in $\mathbb{P}^{2}$, J. Algebra 242 (2001), 720-728. MR 1848967 (2002f:16042)

[10] A. de Jong, The period-index problem for the Brauer group of an algebraic surface, Duke Math. J. 123 (2004), 71-94. MR2060023

[11] B. È. Kunyavskiǔ, L. H. Rowen, S. V. Tikhonov, and V. I. Yanchevskiǔ, Bicyclic algebras of prime exponent over function fields, to appear in Trans. Amer. Math. Soc.

[12] L. H. Rowen, A. S. Sivatski, and J.-P. Tignol, Division algebras over rational function fields in one variable, preprint no. 137 at http://www.mathematik.uni-bielefeld.de/LAG, to appear in Contemp. Math.

[13] D. J. Saltman, Brauer groups of invariant fields, geometrically negligible classes, an equivariant Chow group, and unramified $H^{3}$, "K-Theory and Algebraic Geometry: Connections with Quadratic Forms and Division Algebras" (B. Jacob and A. Rosenberg, Eds.), Proc. Symp. Pure Math., vol. 58, part 1, Amer. Math. Soc., Providence, RI, 1995, pp. 189-246. MR:1327283 (96c:12008)

[14] D. J. Saltman, Lectures on Division Algebras, Amer. Math. Soc., Providence, RI, 1999. MR1692654 (2000f:16023) 
[15] J-P. Serre, Cohomologie galoisienne, 5ème éd., Lecture Notes Math. 5, Springer-Verlag, Berlin et al., 1994. MR.1324577 (96b:12010)

[16] M. Van den Bergh, Division algebras on $\mathbb{P}^{2}$ of odd index ramified along a smooth elliptic curve are cyclic, "Algèbre non commutative, groupes quantiques et invariants (Reims, 1995)", Sémin. Congr., vol. 2, Soc. Math. France, Paris, 1997, pp. 43-53. MR1601190(99d:16021)

Department of Mathematics, Bar-Ilan University, 52900 Ramat Gan, Israel

E-mail address: kunyav@macs.biu.ac.il

Department of Mathematics, Bar-Ilan University, 52900 Ramat Gan, Israel

E-mail address: rowen@macs.biu.ac.il

Institute of Mathematics of the National Academy of Sciences of Belarus, ul. Surganova 11, 220072 Minsk, Belarus

E-mail address: tsv@im.bas-net.by

Institute of Mathematics of the National Academy of Sciences of Belarus, ul. Surganova 11, 220072 Minsk, Belarus

E-mail address: yanch@im.bas-net.by 